\title{
Feasibility of video-assisted thoracoscopic sleeve lobectomy for non-small cell lung cancer
}

\author{
Yeong Jeong Jeon*, Jeonghee Yun ${ }^{\star}$, Yong Soo Choi, Min Soo Kim, Jae Won Choi \\ Department of Thoracic and Cardiovascular Surgery, Samsung Medical Center, Sungkyunkwan University School of Medicine, \\ Seoul, Korea
}

Received: November 26, 2020

Revised: December 9, 2020

Accepted: December 18, 2020

Corresponding author:

Yong Soo Choi

Department of Thoracic

and Cardiovascular Surgery,

Samsung Medical Center,

Sungkyunkwan University

School of Medicine, 81 Irwon-

ro, Gangnam-gu, Seoul 06351, Korea

E-mail: choi.smcts@gmail.com

*Yeong Jeong Jeon and Jeonghee Yun contributed equally to this study as first authors.
Tel: +82-2-3410-1696

\begin{abstract}
Purpose: Sleeve lobectomy is a safe and effective surgical procedure for centrally located non-small cell lung cancer (NSCLC). However, the use of video-assisted thoracoscopic surgery (VATS) on bronchial sleeve resection is still controversial due to a lack of evidence. In this study, we describe our experience on VATS sleeve lobectomy and evaluate its feasibility for treating NSCLC.

Methods: From January 2010 to May 2019, VATS sleeve lobectomy was attempted in 19 patients with NSCLC at Samsung Medical Center. Their baseline characteristics, perioperative data, and survival outcome were collected and analyzed retrospectively.

Results: Of the 19 patients, 10 underwent VATS sleeve lobectomy successfully. The mean age of the patients who underwent successful VATS was $53.5 \pm 15.8$ years, and all the patients were men. Seven patients $(70 \%)$ had squamous cell carcinoma. The mean postoperative chest tube drainage duration was $5.3 \pm 2.3$ days, and the median hospital stay duration was 7 days (interquartile range, 6.25 to 11.5 days). Among the patients who underwent successful VATS, two had postoperative bronchial stenosis: One patient underwent complete pneumonectomy, and one patient was observed without any intervention. During the median follow-up duration of 3.5 years, two patients with squamous cell carcinoma who underwent successful VATS died. Of these, one died 70 days postoperatively because of acute respiratory distress syndrome and one died 18 months postoperatively from an unknown cause. No patient had locoregional recurrence.

Conclusion: VATS sleeve lobectomy is a feasible surgical procedure for centrally located tumors without vascular invasion.
\end{abstract}

Keywords: Carcinoma, non-small-cell lung; Sleeve lobectomy; Thoracic surgery, video-assisted

\section{INTRODUCTION}

distributed under the terms of the Creative Commons Attribution Non-Commercial License (https:// creativecommons.org/licenses/ by-nc/4.0/).
Sleeve lobectomy is a safe and effective surgical option for centrally located non-small cell lung cancer (NSCLC) that not only prevents the risk of incomplete resection of the tumor but also preserves pulmonary function by avoiding pneumonectomy. Many studies have shown 
that sleeve lobectomy is not inferior to pneumonectomy with regard to long-term survival, quality of life, and postoperative risks [1-6]. Thoracotomy has been the standard approach for sleeve bronchial resection due to its complexity and difficulty and the risk of postoperative bronchial complications. However, with increasing experience and technique advances in performing video-assisted thoracoscopic surgery (VATS), the use of VATS is expanding to more complicated cases including sleeve lobectomy. Since the first case report on the use of VATS sleeve lobectomy, published in 2002 [7], it has been used steadily by some thoracic surgeons [811]. Some retrospective studies have shown that VATS sleeve lobectomy is a safe and feasible surgical procedure for treating centrally located NSCLC with comparable perioperative and survival outcomes to the thoracotomy approach [12-16]. However, the application of VATS in bronchial sleeve resection is still controversial due to a lack of evidence.

Thus, in this retrospective study, we describe our experience on VATS sleeve lobectomy and evaluate its feasibility for treating centrally located NSCLC.

\section{METHODS}

\section{Patient selection}

From January 2010 to May 2019, VATS sleeve lobectomy was attempted in 19 patients with NSCLC by a single surgeon at Samsung Medical Center. Data associated with the patients' baseline characteristics, operative details, perioperative course, survival, and recurrence were collected from the medical records. Postoperative pathological staging was performed according to the eighth edition of the tumor-nodemetastasis (TNM) classification by the International Association for the Study of Lung Cancer.

For the preoperative evaluation, physical examination, chest radiography, contrast-enhanced chest computed tomography (CT), positron emission tomography, and pulmonary function tests were performed in all patients. Moreover, bronchoscopic evaluation was performed when the tumor was suspected to invade the bronchus. For mediastinal nodal staging, mediastinoscopy or endobronchial ultrasoundguided transbronchial needle biopsy was performed. If N2 disease was confirmed by biopsy, the patient underwent neoadjuvant chemoradiation therapy followed by surgery. Adjuvant therapy was recommended for patients with pathologic stage II or III NSCLC or positive resection margin. After surgery, patients regularly visited the hospital for recurrence surveillance. Chest CT was performed every 3 to 6 months.
The study was approved by the Institutional Review Board (IRB No. 2020-11-120) of Samsung Medical Center, and informed consent was waived.

\section{Surgical procedure}

General anesthesia with one-lung ventilation was induced using a double-lumen endotracheal tube. The patients were placed in a lateral decubitus position. For the VATS approach, a utility port was placed in the fifth intercostal space (ICS) and one or two additional ports were made in the seventh or fourth ICS as needed. The surgeon operated on the right side of the operating table regardless of patient positioning (either left or right lateral decubitus). An assistant using a thoracoscope routinely stood opposite the surgeon and rotated the camera head $90^{\circ}$ toward the surgeon. This positioning provided the assistants a view consistent with conventional thoracotomy. The surgeon's monitor was rotated $180^{\circ}$ (upside down) to provide the same orientation as seen in open thoracotomy. This setting of orientation and monitoring system provided good ergonomics and comfortable handling (Fig. 1).

For the bronchial sleeve resection, the main bronchus and lobar bronchus were skeletonized after dissecting the lymph nodes. The main bronchus and bronchus of the reserved lobe were cut off $1 \mathrm{~cm}$ from the tumor margin. After the negative margins were confirmed on frozen biopsy, bronchial

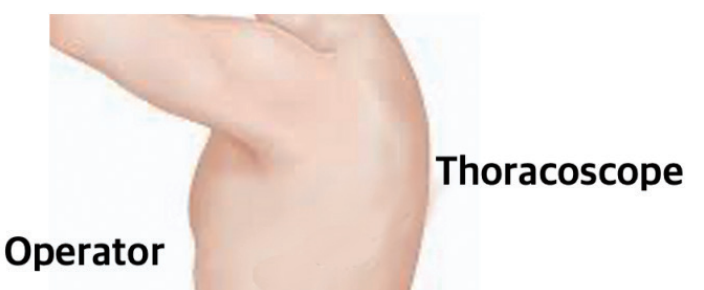

Assistance

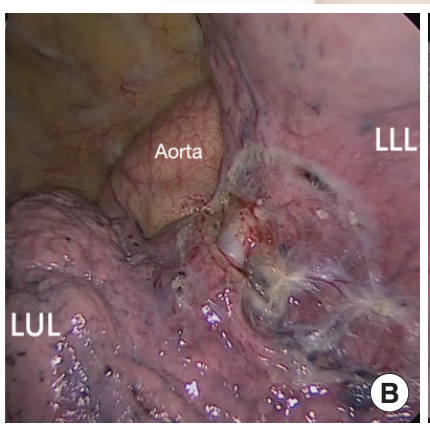

(A)

Fig. 1. Video-assisted thoracoscopic surgery setting for left side approach (A), operator' view (B), and assistant's view (C). LUL, left upper lobe; LLL, left lower lobe. 
PRECISION AND FUTURE MEDICINE

Yeong Jeong Jeon, et al.

anastomosis was performed. Simple interrupted sutures with three or four inner knots or simple continuous sutures were placed in the posterior side; then, multiple outer knots were placed in the anterior and lateral sides. Fibrin sealant was applied to the anastomosis site for reinforcement. After the bronchial anastomosis, an air leakage test of the bronchus was conducted using normal saline to confirm that no air leakage was present under $20 \mathrm{~cm} \mathrm{H}_{2} \mathrm{O}$ airway pressure.

\section{Statistical analysis}

The patients' baseline characteristics and perioperative outcomes including the number of resected lymph nodes, operating time, duration of intensive care unit and hospital stay, and duration of postoperative chest tube drainage were analyzed using SPSS version 25 (IBM Corp., Armonk, NY, USA). The measurement data were expressed as mean \pm standard deviations. The overall and recurrence-free survival rates were evaluated using Kaplan-Meier curve analysis.

\section{RESULTS}

\section{Patient characteristics}

The baseline characteristics of the 10 patients who underwent successful VATS sleeve lobectomy are described in Table 1 . The mean age of the patients undergoing successful VATS was $53.5 \pm 15.8$ years, and all patients were male. The patients who underwent successful VATS had tumors located in the left upper $(n=6)$, left lower $(n=3)$, and right upper lobes $(n=1)$. In addition, the clinical TNM staging were $T 1(n=3), T 2$ $(n=7), N 0(n=6), N 1(n=3)$, and N2 ( $n=1)$.

The characteristics of the 19 patients for whom VATS sleeve lobectomy was attempted are shown in Supplementary Table 1 . The mean age of the patients was $59.7 \pm 14$ years, and most patients (89.5\%) were male. Tumors were located in the left upper ( $n=12)$, right upper $(n=4)$, and left lower lobes $(n=3)$. The clinical TNM staging was as follows: T1 $(n=3$, $15.8 \%), \mathrm{T} 2(\mathrm{n}=15,78.9 \%)$, and T3 $(\mathrm{n}=1,5 \%)$ and NO $(\mathrm{n}=11$, $57.9 \%), \mathrm{N} 1(\mathrm{n}=7,36.8 \%)$, and N2 ( $\mathrm{n}=1,5 \%)$. No patient had a history of receiving neoadjuvant treatments.

\section{Surgical outcomes}

Among 19 patients for whom VATS was attempted, nine patients (47.4\%) were converted to thoracotomy. The main reason for the conversion was tumor invasion to the main pulmonary artery that required vascular sleeve operation $(n=5)$. Other reasons were failure of the one-lung ventilation $(n=2)$, anthracofibrotic lymph node $(n=1)$, and insufficient resec-
Table 1. Characteristics of patients undergoing VATS sleeve lobectomy

\begin{tabular}{|c|c|}
\hline Characteristic & Successful VATS $(n=10)$ \\
\hline Age (yr) & $53.5 \pm 15.8$ \\
\hline Male sex & $10(100)$ \\
\hline \multicolumn{2}{|l|}{ Smoking history } \\
\hline Never smoker & $1(10)$ \\
\hline Ex-smoker & $4(40)$ \\
\hline Current smoker & $5(50)$ \\
\hline \multicolumn{2}{|l|}{ Pulmonary function test } \\
\hline FEV1 (\%PRED) & $84.9 \pm 10.3$ \\
\hline DLCO (\%PRED) & $85.6 \pm 15$ \\
\hline \multicolumn{2}{|l|}{ Comorbidities } \\
\hline Diabetes & $2(20)$ \\
\hline Hypertension & $1(10)$ \\
\hline Chronic lung disease & $2(20)$ \\
\hline Cerebrovascular disease & $2(20)$ \\
\hline Chronic liver disease & $2(20)$ \\
\hline Previous malignancy & 0 \\
\hline \multicolumn{2}{|l|}{$\mathrm{CCl}$ score } \\
\hline 0 & $2(20)$ \\
\hline 1 & $7(70)$ \\
\hline 2 & 0 \\
\hline$\geq 3$ & $1(10)$ \\
\hline \multicolumn{2}{|l|}{ Tumor location } \\
\hline RUL & $1(10)$ \\
\hline LUL & $6(60)$ \\
\hline LLL & $3(30)$ \\
\hline \multicolumn{2}{|l|}{ Clinical T stage } \\
\hline $\mathrm{T} 1$ & $3(30)$ \\
\hline $\mathrm{T} 2$ & $7(70)$ \\
\hline $\mathrm{T} 3$ & 0 \\
\hline \multicolumn{2}{|l|}{ Clinical N stage } \\
\hline No & $6(60)$ \\
\hline $\mathrm{N} 1$ & $3(30)$ \\
\hline N2 & $1(10)$ \\
\hline
\end{tabular}

Values are presented as mean \pm standard deviation or number (\%). VATS, video-assisted thoracoscopic surgery; FEV1, forced expiratory volume in 1 second; PRED, predicted; DLCO, diffusing capacity for carbon monoxide; $\mathrm{CCl}$, Charlson comorbidity index; RUL, right upper lobe; LUL, left upper lobe; LLL, left lower lobe.

tion margin $(n=1)$. Postoperative pathological examination indicated that of the patients who underwent successful VATS: seven had squamous cell carcinoma, two had mucoepidermoid carcinoma, one had atypical carcinoid tumor. 
PRECISION AND FUTURE MEDICINE

VATS sleeve lobectomy

Table 2. Perioperative outcomes of VATS sleeve lobectomy

\begin{tabular}{|c|c|}
\hline Variable & Successful VATS $(n=10)$ \\
\hline \multicolumn{2}{|l|}{ Histology } \\
\hline Squamous cell carcinoma & $7(70)$ \\
\hline Mucoepidermoid carcinoma & $2(20)$ \\
\hline Atypical carcinoid tumor & $1(10)$ \\
\hline Tumor size (cm) & $2.6 \pm 1.4$ \\
\hline \multicolumn{2}{|l|}{ Pathologic T stage } \\
\hline $\mathrm{T} 1$ & $5(50)$ \\
\hline $\mathrm{T} 2$ & $5(50)$ \\
\hline T3 & 0 \\
\hline \multicolumn{2}{|l|}{ Pathologic N stage } \\
\hline NO & $6(60)$ \\
\hline N1 & $4(40)$ \\
\hline N2 & 0 \\
\hline Lymphatic invasion & $3(30)$ \\
\hline Vascular invasion & 0 \\
\hline Perineural invasion & $2(20)$ \\
\hline \multicolumn{2}{|l|}{ Adjuvant therapy } \\
\hline Concurrent chemoradiation & $1(10)$ \\
\hline Chemotherapy & $3(30)$ \\
\hline $\mathrm{R} 0$ resection & $10(100)$ \\
\hline Bronchial margins $(\mathrm{cm})$ & $0.7 \pm 0.5$ \\
\hline Total resected lymph nodes & $16.7 \pm 7.9$ \\
\hline Positive lymph nodes & $0.8 \pm 1.3$ \\
\hline Operating time (min) & $236 \pm 79$ \\
\hline Intensive care unit stay (day) & $2 \pm 2.5$ \\
\hline Chest tube duration (day) & $5.3 \pm 2.3$ \\
\hline Postoperative hospital stay (day) & $7(6.25-11.5)$ \\
\hline
\end{tabular}

Values are presented as number (\%), mean \pm standard deviation, or median (interquartile range).

VATS, video-assisted thoracoscopic surgery.

Furthermore, in these patients, the mean operative duration was $236 \pm 79$ minutes from the time of skin incision to wound closure, and the mean number of lymph nodes dissected was $16.7 \pm 7.9$ per patient. In addition, complete resection was achieved in all patients, and the mean length of bronchial resection margin was $0.7 \pm 0.5 \mathrm{~cm}$ in patients with successful VATS.

In patients who underwent VATS successfully, the mean postoperative chest tube indwelling time was $5.3 \pm 2.3$ days, and the median hospital stay duration was 7 days (interquartile range, 6.25 to 11.5 days). Pathological and operative data have been summarized in Table 2. Two patients had postop-
Table 3. Perioperative complications

\begin{tabular}{ll}
\hline Variable & Successful VATS $(\mathbf{n}=10)$ \\
\hline Pneumonia & $1(10)$ \\
Acute respiratory distress syndrome & $1(10)$ \\
Postoperative bronchial stenosis & $2(20)$ \\
Prolonged air-leak & 0 \\
Empyema & 0 \\
Atrial fibrillation & $1(10)$ \\
Vocal code palsy & 0
\end{tabular}

Values are presented as number (\%).

VATS, video-assisted thoracoscopic surgery.

Table 4. Pattern of recurrence

\begin{tabular}{lc}
\hline Variable & Successful VATS $(\mathrm{n}=10)$ \\
\hline Locoregional & 0 \\
Bronchial anastomosis site & 0 \\
Ipsilateral lymph node & 0 \\
Distant & $2(20)$ \\
\hline
\end{tabular}

Values are presented as number (\%).

VATS, video-assisted thoracoscopic surgery.

erative bronchial stenosis. One of these underwent completion pneumonectomy, and one was observed without any intervention. The postoperative complications that were observed in the cohort are shown in Table 3.

The perioperative outcomes and complications of patients for whom VATS sleeve lobectomy was attempted are described in Supplementary Tables 2, 3.

\section{Survival outcomes}

No patients had died perioperatively. The median follow-up duration was 3.5 years. Among seven patients with squamous cell carcinoma who underwent successful VATS, two died. One patient died 70 days postoperatively because of acute respiratory distress syndrome, and the other died 18 months after the surgery from an unknown cause. No patient had locoregional recurrence (Table 4).

The pattern of recurrence of patients for whom VATS was attempted are also shown in Supplementary Table 4.

\section{DISCUSSION}

The VATS approach has been widely adopted in treating NSCLC. Compared to open thoracotomy, VATS had many advantages including reduced postoperative pain, preserved 
lung function, reduced hospital stay duration, and reduced postoperative chest tube drainage duration [17-19]. Nevertheless, the use of VATS in patients with centrally located lung cancer who need sleeve bronchial resection is still controversial because of oncological efficacy and because it is technically demanding. With technological advances in surgical instruments, high-resolution cameras, and three-dimensional systems, several authors have successfully performed VATS sleeve lobectomy [20-23].

This study evaluated the feasibility and oncological outcome of VATS sleeve lobectomy. In terms of postoperative outcomes, including the duration of chest tube drainage, hospital stay duration, and incidence of complications, the outcomes of this study conform to the literature on conventional thoracotomy [12,24]. Recently, Gao et al. [12] have reported that VATS sleeve lobectomy was associated with less blood loss, shorter chest tube drainage duration, and shorter postoperative hospital stay duration. Moreover, Yang et al. [14] have shown that patients who underwent VATS sleeve lobectomy had a lower incidence of postoperative complications and lesser duration of postoperative thoracic drainage.

Regarding oncological results, in this study, the 5-year overall survival rate of all patients who underwent successful VATS was $80 \%$, whereas that of patients with squamous cell carcinoma was $71.4 \%$. Furthermore, the 5 -year recurrence-free survival rate of patients with squamous cell carcinoma was $40 \%$. The main concern about the use of VATS in sleeve bronchial resection is local recurrence. This study showed that the local recurrence rate after VATS sleeve resection was $0 \%$. These survival and recurrence outcome were comparable to the outcomes of conventional sleeve lobectomy in the literature $[1,5,12,14,15,24,25]$. In 2013, Kasprzyk et al. [26] have reported that the 5 -year survival rate after conventional sleeve lobectomy was $56.1 \%$, and the local recurrence rate was 9.3\%. In 2009, Merritt et al. [24] have shown that the 5 -year survival rates for patients who underwent conventional sleeve lobectomy for pathological N0 and N1 NSCLC were $52.6 \%$ and $39.3 \%$, respectively. Furthermore, in 2015, Zhou et al. [13] have demonstrated that the overall 1-, 3-, and 4-year survival rates of patients who underwent VATS sleeve lobectomy were 100\%, 73\%, and $40 \%$, respectively. For those who underwent thoracotomy, the overall 1-, 3-, and 4-year survival rates were $100 \%, 63 \%$, and $56 \%$, respectively.

The rate of conversion to thoracotomy was high (9/19, $47.4 \%$ ) in this study. The main reason for the conversion was tumor invasion to the main pulmonary artery requiring vas- cular sleeve operation (5/9,55.6\%). When the vascular sleeve procedure was needed, we preferred to convert to thoracotomy for safe and accurate anastomosis of the vessels. In the VATS approach, there is generally limited space for instrumentation to allow clamping and anastomosis of the main pulmonary artery; thus, operation time is much increased. Prolonged duration for main pulmonary artery clamping and anastomosis may cause ischemia-reperfusion injury or interstitial/alveolar pulmonary edema. If tumor invasion to the main pulmonary artery is strongly suspected on preoperative imaging studies, immediate thoracotomy rather than VATS may reduce the conversion rate to thoracotomy in this series.

The operative time of VATS sleeve lobectomy was long, probably due to the more challenging and complicated operative techniques associated with VATS. During thoracoscopic sleeve lobectomy, one of the technical challenges is related to bronchial anastomosis. We traditionally have applied simple interrupted sutures with three or four inner knots for the membranous part of the bronchus and multiple outer knots for the cartilage part. Recently, we applied simple continuous sutures for the membranous part combined with simple interrupted sutures for the cartilage part. The change from interrupted sutures to continuous sutures for the membranous part shortened the operative time.

This study has several limitations. First, it was a retrospective study and there could be a treatment selection bias. Although VATS sleeve lobectomy achieved outcomes comparable to those of open surgery in this study, the selection bias for VATS sleeve lobectomy may have improved the outcome. Second, the information for VATS indication is insufficient. In addition, it included a relatively small number of patients from an experienced surgeon. VATS was performed only in few patients because of the relatively narrow indication of VATS for sleeve lobectomy. If our experience accumulates, the inclusion criteria could be expanded. Third, this was a single-arm study with a relatively short-term follow-up. Future prospective studies that compare VATS with open thoracotomy need to be conducted to validate the efficacy of VATS.

Despite these limitations, we showed that the outcomes of VATS sleeve lobectomy were comparable to those of conventional sleeve lobectomy that have been document in the literature. Our surgical technique and real-world practice about VATS sleeve lobectomy are informative for most surgeons who perform minimally invasive procedures.

In conclusion, performing sleeve lobectomy using the VATS approach is feasible and safe without compromising the on- 
cological efficacy. In this series, the main reason for conversion to thoracotomy was tumor invasion to the main pulmonary artery that necessitated vascular sleeve operation. If vascular invasion of the tumor is strongly suspected preoperatively, planning thoracotomy rather than VATS may reduce the conversion rate to thoracotomy.

\section{CONFLICTS OF INTEREST}

No potential conflict of interest relevant to this article was reported.

\section{ORCID}

Yeong Jeong Jeon

Jeonghee Yun

Yong Soo Choi

Min Soo Kim

Jae Won Choi https://orcid.org/0000-0001-6745-6131

https://orcid.org/0000-0002-3774-9280

https://orcid.org/0000-0001-8492-7644

https://orcid.org/0000-0002-9069-619X

https://orcid.org/0000-0003-3984-0296

\section{AUTHOR CONTRIBUTIONS}

Conception or design: JY, YSC.

Acquisition, analysis, or interpretation of data: YJJ, JY, YSC, MSK, JWC.

Drafting the work or revising: YJJ, JY, YSC.

Final approval of the manuscript: YJJ, JY, YSC, MSK, JWC.

\section{REFERENCES}

1. Deslauriers J, Gregoire J, Jacques LF, Piraux M, Guojin L, Lacasse Y. Sleeve lobectomy versus pneumonectomy for lung cancer: a comparative analysis of survival and sites or recurrences. Ann Thorac Surg 2004;77:1152-6.

2. Ludwig C, Stoelben E, Olschewski M, Hasse J. Comparison of morbidity, 30-day mortality, and long-term survival after pneumonectomy and sleeve lobectomy for nonsmall cell lung carcinoma. Ann Thorac Surg 2005;79:96873.

3. Ma Z, Dong A, Fan J, Cheng H. Does sleeve lobectomy concomitant with or without pulmonary artery reconstruction (double sleeve) have favorable results for nonsmall cell lung cancer compared with pneumonectomy?: a meta-analysis. Eur J Cardiothorac Surg 2007;32:20-8.

4. Schirren J, Bolukbas S, Bergmann T, Fisseler-Eckhoff A, Trainer S, Beqiri S. Prospective study on perioperative risks and functional results in bronchial and bronchovas- cular sleeve resections. Thorac Cardiovasc Surg 2009;57: 35-41.

5. Takeda S, Maeda H, Koma M, Matsubara Y, Sawabata N, Inoue $\mathrm{M}$, et al. Comparison of surgical results after pneumonectomy and sleeve lobectomy for non-small cell lung cancer: trends over time and 20-year institutional experience. Eur J Cardiothorac Surg 2006;29:276-80.

6. Yildizeli B, Fadel E, Mussot S, Fabre D, Chataigner O, Dartevelle PG. Morbidity, mortality, and long-term survival after sleeve lobectomy for non-small cell lung cancer. Eur J Cardiothorac Surg 2007;31:95-102.

7. Santambrogio L, Cioffi U, De Simone M, Rosso L, Ferrero S, Giunta A. Video-assisted sleeve lobectomy for mucoepidermoid carcinoma of the left lower lobar bronchus: a case report. Chest 2002;121:635-6.

8. Agasthian T. Initial experience with video-assisted thoracoscopic bronchoplasty. Eur J Cardiothorac Surg 2013; 44:616-23.

9. Chen H, Huang L, Xu G, Zheng B, Zheng W, Zhu Y, et al. Modified bronchial anastomosis in video-assisted thoracoscopic sleeve lobectomy: a report of 32 cases. J Thorac Dis 2016;8:2233-40.

10. Chen H, Xu G, Zheng B, Zheng W, Zhu Y, Guo Z, et al. Initial experience of single-port video-assisted thoracoscopic surgery sleeve lobectomy and systematic mediastinal lymphadenectomy for non-small-cell lung cancer. J Thorac Dis 2016;8:2196-202.

11. Davoli F, Bertolaccini L, Pardolesi A, Solli P. Video-assisted thoracoscopic surgery bronchial sleeve lobectomy. J Vis Surg 2017;3:41.

12. Gao HJ, Jiang ZH, Gong L, Ma K, Ren P, Yu ZT, et al. Video-assisted vs thoracotomy sleeve lobectomy for lung cancer: a propensity matched analysis. Ann Thorac Surg 2019;108:1072-9.

13. Zhou S, Pei G, Han Y, Yu D, Song X, Li Y, et al. Sleeve lobectomy by video-assisted thoracic surgery versus thoracotomy for non-small cell lung cancer. J Cardiothorac Surg 2015;10:116.

14. Yang Y, Mei J, Lin F, Pu Q, Ma L, Liu C, et al. Comparison of the short- and long-term outcomes of video-assisted thoracoscopic surgery versus open thoracotomy bronchial sleeve lobectomy for central lung cancer: a retrospective propensity score matched cohort study. Ann Surg Oncol 2020;27:4384-93.

15. Mayne NR, Darling AJ, Raman V, Balderson S, Berry MF, Harpole DH Jr, et al. Perioperative outcomes and 5-year survival after open versus thoracoscopic sleeve resection 
for lung cancer. Semin Thorac Cardiovasc Surg 2020 Aug 25 [Epub]. https://doi.org/10.1053/j.semtcvs.2020.08.013.

16. Zhong Y, Wang Y, Hu X, Wang G, She Y, Deng J, et al. A systematic review and meta-analysis of thoracoscopic versus thoracotomy sleeve lobectomy. J Thorac Dis 2020;12: 5678-90.

17. Stephens N, Rice D, Correa A, Hoffstetter W, Mehran R, Roth J, et al. Thoracoscopic lobectomy is associated with improved short-term and equivalent oncological outcomes compared with open lobectomy for clinical Stage I non-small-cell lung cancer: a propensity-matched analysis of 963 cases. Eur J Cardiothorac Surg 2014;46:60713.

18. Bendixen M, Jorgensen OD, Kronborg C, Andersen C, Licht PB. Postoperative pain and quality of life after lobectomy via video-assisted thoracoscopic surgery or anterolateral thoracotomy for early stage lung cancer: a randomized controlled trial. Lancet Oncol 2016;17:83644.

19. Valo JK, Kyto V, Sipila J, Rautava P, Sihvo E, Gunn J. Thoracoscopic surgery for lung cancer is associated with improved survival and shortened admission length: a nationwide propensity-matched study. Eur J Cardiothorac Surg 2020;57:100-6.

20. Gonzalez-Rivas D, Yang Y, Sekhniaidze D, Stupnik T, Fernandez R, Lei J, et al. Uniportal video-assisted thoraco- scopic bronchoplastic and carinal sleeve procedures. J Thorac Dis 2016;8(Suppl 2):S210-22.

21. Kim TH, Hong TH, Choi YS. Comparison of surgical outcomes between 3D and 2D VATS lobectomy for clinical stage I lung cancer. Precis Future Med 2020;4:69-74.

22. Gonzalez-Rivas D, Garcia A, Chen C, Yang Y, Jiang L, Sekhniaidze $D$, et al. Technical aspects of uniportal video-assisted thoracoscopic double sleeve bronchovascular resections. Eur J Cardiothorac Surg 2020;58(Supplement_ 1):i14-22.

23. Soultanis KM, Chen Chao M, Chen J, Wu L, Yang C, Gonzalez-Rivas D, et al. Technique and outcomes of 79 consecutive uniportal video-assisted sleeve lobectomies. Eur J Cardiothorac Surg 2019;56:876-82.

24. Merritt RE, Mathisen DJ, Wain JC, Gaissert HA, Donahue D, Lanuti M, et al. Long-term results of sleeve lobectomy in the management of non-small cell lung carcinoma and low-grade neoplasms. Ann Thorac Surg 2009;88:1574-82.

25. Li Y, Wang J. Video-assisted thoracoscopic surgery sleeve lobectomy with bronchoplasty. World J Surg 2013;37:16615.

26. Kasprzyk M, Dyszkiewicz W, Piwkowski C, Brudlo M, Galecki B, Gabryel P, et al. Sleeve lobectomy as an alternative to pneumonectomy in patients with operable lung cancer. Neoplasma 2013;60:62-7. 